\title{
BMJ Open Association among the exposure to giving knowledge, attitude and practice for handwashing in people residing in Seoul, South Korea: a retrospective cross-sectional study
}

Jayeun Kim (D) , ${ }^{1,2}$ Kyuhyun Yoon (D) ${ }^{1}$

To cite: Kim J, Yoon K. Association among the exposure to giving knowledge, attitude and practice for handwashing in people residing in Seoul, South Korea: a retrospective crosssectional study. BMJ Open 2022;12:e053329. doi:10.1136/ bmjopen-2021-053329

- Prepublication history and additional supplemental material for this paper are available online. To view these files, please visit the journal online (http://dx.doi.org/10.1136/ bmjopen-2021-053329).

Received 12 May 2021 Accepted 25 January 2022

Check for updates

(C) Author(s) (or their employer(s)) 2022. Re-use permitted under CC BY-NC. No commercial re-use. See rights and permissions. Published by BMJ.

${ }^{1}$ Institute of Health and Environment, Seoul National University, Gwanak-Gu, Seoul, The Republic of Korea

${ }^{2}$ Korea Institute of Child Care and Education, Jung-gu, Seoul, Republic of Korea

Correspondence to

Dr Kyuhyun Yoon;

swnknh@snu.ac.kr

\section{ABSTRACT}

Objectives Handwashing is known as the most preventive method for various infectious diseases. Health authorities have conducted various campaigns and public relations targeting the general population but few evaluations of these long-term interventions. This study aimed to investigate the association between experience of educational events or public relations (EXEP), attitude towards the effectiveness of handwashing (AEHW) and handwashing practice (HWP).

Setting Using a population-based cross-sectional study with a complex sample design, we explored the response outcomes of handwashing. Those outcomes were collected biennially using Community Health Survey among the years 2013, 2015, 2017 and 2019. We applied multivariate logistic regression for the association among sociodemographic factors, health conditions, EXEP, AEHW and HWP.

Participants Total participants residing in Seoul were 23139 (men 44.2\%), 23004 (men 44.4\%), 22955 (men 44.8\%) and 22930 (men 43.9\%), in 2013, 2015, 2017 and 2019, respectively.

Results Overall, participants with EXEP are more likely to be women, aged between the 30s and 60s, and with a higher educational level. Particularly, participants with EXEP had an increased association with HWP (OR 1.21, $95 \% \mathrm{Cl} 1.19$ to 1.33 ) and AEHW (OR 1.50, 95\% Cl 1.38 to 1.63). In addition, AEHW also had an increased association with HWP (OR 1.67, 95\% $\mathrm{Cl} 1.53$ to 1.81). The mediated effect of AEHW to HWP is not determined evidently. Conclusion The results showed that EXEP is positively associated with HWP and AEHW among communitydwelling people. Our results confirm that educational activity can lead to more pronounced behavioural changes only when it forms a positive attitude.

\section{INTRODUCTION}

Handwashing is widely accepted as a primary method to prevent infectious disease by dismissing the spread of pathogens. ${ }^{1-6}$ Occupational handwashing came to be emphasised by WHO and other health authorities because contaminated hands of healthcare
Strengths and limitations of this study

- We applied Knowledge, Attitude and Practice theory to evaluate handwashing effectiveness for the community-based interventions.

- We found consistent outcome that the experience of educational events or public relations raised a positive attitude towards the effectiveness of handwashing and handwashing practice in daily life among the community-dwelling people.

- Although the association was varied according to sociodemographics and health status, the participants who believed in the preventive effect of handwashing on infectious diseases were more likely to practice handwashing frequently in their daily life.

- Educational activity related to preventive behaviour is likely to lead to more pronounced behavioural changes when accompanied by a positive attitude and belief of its effectiveness.

providers are a primary source of pathogenic spread. $^{3-5}$ Hand hygiene is the single most important practice in the reduction of the transmission of infection among workers in the healthcare setting.

Furthermore, hand hygiene is a vital method to apply non-pharmaceutical interventions. An open-label randomised study conducted in the UK suggested that an internet-delivered handwashing intervention reduced the number and severity of respiratory tract infections among both the participants and their households. ${ }^{6}$

Korea Disease Control and Prevention Agency (KCDC (predecessor of KDCA) had conducted two surveys for handwashing. The one was national education programmes and publicity campaigns through the National Handwashing Campaign Center from 2005 to 2012 and four awareness surveys carried out from 2006 to 2011 to evaluate the 
programmes. The other is the 2013-2014 National Handwashing Surveys. ${ }^{7}$ As a result of the national awareness survey, participants answered that they used soap and detergent to wash their hands an average of 6.6 times a day among adults. That was a good increase from 4.8 in $2006 .{ }^{8}$ Then, handwashing was strongly recommended as a self-hygiene application during pandemics of respiratory infectious disease such as H1N1 influenza ${ }^{9}$ or Middle East Respiratory Syndrome (MERS) ${ }^{10} 11$ in Korea. During the pandemic of H1N1 influenza in 2009, WHO recommended handwashing as a self-preventive method. ${ }^{3}{ }^{12}$ MERS pandemic occurred in South Korea in 2015 and handwashing was strongly emphasised in terms of a selfpreventive method. ${ }^{10}{ }^{11} 13$ Even so, in terms of community health promotion, its effectiveness has not been fully investigated. Most studies regarding the effectiveness of handwashing are centred on children, mostly within a school environment ${ }^{14-16}$ or low-income and middleincome countries. $^{17}$

Therefore, we studied whether the experience of educational events or public relations (EXEP) raised a positive attitude towards the effectiveness of handwashing (AEHW) and handwashing practice (HWP) in daily life among the community-dwelling people. A series of questions related to handwashing is based on the 'Knowledge, Attitude and Practice (KAP) theory'.

The survey with KAP theory figures out knowledge, attitudes and practices on a specific topic and is useful to plan or evaluate interventions in the community. In general, a KAP survey is repeatedly conducted before and after a project and is used to evaluate its effectiveness. In the KAP model, knowledge goes beyond simply knowing to refer to the acquisition, maintenance and use of information and technology. ${ }^{18}$ Attitude is an acquired element of each individual and includes all of cognition, affect, sense and behavioural tendency. ${ }^{19}$ Those three pillars are connected to each other. However, when evaluating interventions (mainly educational programmes for a behavioural change) to bring about changes in health behaviour, we can build a logic model of the results by arranging these three elements in stages. Accordingly, we set the logic model considering the hierarchy of the outcomes among EXEP, AEHW and HWP.

This study aimed to investigate the association between experience of EXEP, AEHW and HWP.

\section{MATERIALS AND METHODS Study design}

Community Health Survey (CHS) is a population-based cross-sectional survey with a complex sample design. CHS data provide individual-level information on health status and its determinants by the municipal community. ${ }^{20}$ $\mathrm{KCDA}$, in conjunction with public health centres, began periodically CHS in 2008. Nationwide participants $(\geq 19$ years) were sampled with stratification of community and house type. The data are collected using computerassisted personal interviews. For the personal interview, the survey questionnaire is asked to participants. The reference period of questions varies from a week to a year.

\section{Study population and setting}

Among the nationwide participants ( $\geq 19$ years) of CHS collected under the complex sample design, study participants for this study are restricted to adults residing in Seoul. The purpose of participants' restriction is due to social consistency and demographic homogeneity for the entire study period. Accordingly, approximately 23 000 participants were recruited per survey. The personal hygiene section including handwashing was introduced since 2013 and the personal hygiene section is surveyed biennially with retrospective questionnaire using reference period such as within a week or a year. Consequently, our study used data from the years 2013, 2015, 2017 and 2019 to differentiate daily HWP for the adult residents in Seoul.

\section{Measures}

The questionnaire for handwashing includes one subjective question of the AEHW to prevent infectious diseases and four questions regarding behavioural practice in handwashing (online supplemental figure S1). This study recruited EXEP, AEHW and HWP with soap or detergents as response variables. We defined EXEP using the following question; "In the past year, have you experienced any education on proper hand-washing or any related promotional materials?" Although the experiences of education and public relations are different, the question itself was designed to collect both information at once (online supplemental figure S1).

Among the dependent variables, frequency of HWP was categorised into two groups, 'frequently and more' and the other with 'occasionally', 'rarely' or 'never'. The reference group was the populations with less practice.

Explanatory variables were sex, age, education, comorbidity conditions, self-rated health and oral health status. Age was categorised by 20s including 19 years old, 30s, $40 \mathrm{~s}, 50 \mathrm{~s}, 60 \mathrm{~s}, 70 \mathrm{~s}$ and 80 and more. Education was tiered with college or upper, middle-high school and elementary school or lower. Several comorbidity conditions were surveyed annually, but the diseases were various. Comorbidity conditions were grouped with allergic or respiratory diseases, and other chronic conditions with hypertension, diabetes mellitus, dyslipidaemia or arthritis. Self-rated health status and oral health status were given five scales: very good, good, usual, poor and very poor. We re-categorised those self-ratings into good+, usual and poor-.

\section{Statistical analysis}

The CHS data have regional strata and individual sampling weight based on the number of household members and age-stratified population. Thus, we analysed the data with sampling weight adjustment in order to examine the relationship between explanatory variables and handwashing-related outcomes. In other words, the design effect of clustering was accounted and weight was 
Table 1 Descriptive statistics of handwashing-related outcomes in Community Health Survey in 2013, 2015, 2017 and 2019 in Seoul, South Korea

\begin{tabular}{|c|c|c|c|c|c|}
\hline \multirow{2}{*}{ Variables } & & \multicolumn{4}{|l|}{ Year, n (\%) } \\
\hline & & 2013 & 2015 & 2017 & 2019 \\
\hline \multicolumn{2}{|l|}{ Total participants } & 23139 (100.0) & 23004 (100.0) & 22955 (100.0) & 22930 (100.0) \\
\hline \multirow[t]{2}{*}{ Sex } & Male & $10231(44.2)$ & $10218(44.4)$ & 10278 (44.8) & 10064 (43.9) \\
\hline & Female & $12908(55.8)$ & $12786(55.6)$ & 12677 (55.2) & 12866 (56.1) \\
\hline \multirow[t]{7}{*}{ Age (years) } & $19-29$ & 3828 (16.5) & $3760(16.3)$ & $3655(15.9)$ & $3363(14.7)$ \\
\hline & 30-39 & $4493(19.4)$ & 4189 (18.2) & $3851(16.8)$ & $3442(15.0)$ \\
\hline & $40-49$ & 4687 (20.3) & 4515 (19.6) & 4428 (19.3) & 3810 (16.6) \\
\hline & $50-59$ & 4440 (19.2) & 4239 (18.4) & $4288(18.7)$ & 4168 (18.2) \\
\hline & $60-69$ & 3114 (13.5) & 3437 (14.9) & 3540 (15.4) & 4054 (17.7) \\
\hline & $70-79$ & $2053(8.9)$ & $2182(9.5)$ & $2376(10.4)$ & 2941 (12.8) \\
\hline & $80+$ & $524(2.3)$ & $682(3.0)$ & $817(3.6)$ & $1152(5.0)$ \\
\hline \multirow[t]{3}{*}{ Education } & College+ & 12067 (52.2) & $12411(54.0)$ & 12531 (54.6) & 11907 (51.9) \\
\hline & Middle-high & $8355(36.1)$ & $8002(34.8)$ & $7966(34.7)$ & $8261(36.0)$ \\
\hline & Elementary- & 2667 (11.5) & $2516(10.9)$ & $2416(10.5)$ & $2723(11.9)$ \\
\hline Chronic conditions $1^{*}$ & Yes & $5678(24.5)$ & $5923(25.7)$ & $6566(28.6)$ & $6183(27.0)$ \\
\hline Chronic conditions $2 \dagger$ & Yes & $1119(4.8)$ & $1167(5.1)$ & $709(3.1)$ & - \\
\hline \multirow[t]{3}{*}{ Self-rated health } & Good+ & 9801 (42.4) & $10036(43.6)$ & $10063(43.8)$ & 9010 (39.3) \\
\hline & Usual & $10104(43.7)$ & $9819(42.7)$ & 9697 (42.2) & $10392(45.3)$ \\
\hline & Poor- & 3233 (14.0) & 3147 (13.7) & 3193 (13.9) & $3524(15.4)$ \\
\hline \multirow[t]{3}{*}{ Self-rated oral health } & Good+ & $6267(27.1)$ & $6253(27.2)$ & 6635 (28.9) & $6203(27.1)$ \\
\hline & Usual & 9685 (41.9) & 9788 (42.5) & 9995 (43.5) & $10360(45.2)$ \\
\hline & Poor- & 7186 (31.1) & 6961 (30.3) & $6324(27.5)$ & $6364(27.8)$ \\
\hline $\begin{array}{l}\text { Experience of educational } \\
\text { events or public relations }\end{array}$ & Yes & $14922(64.5)$ & $18491(80.4)$ & $16141(70.3)$ & $15538(67.8)$ \\
\hline $\begin{array}{l}\text { Attitude of the preventive } \\
\text { effect }\end{array}$ & $\begin{array}{l}\text { Very helpful or } \\
\text { helpful }\end{array}$ & 22926 (99.1) & 22662 (98.5) & 22791 (99.3) & 22753 (99.2) \\
\hline $\begin{array}{l}\text { Practice with soap or hand } \\
\text { detergents }\end{array}$ & $\begin{array}{l}\text { Always or } \\
\text { frequently }\end{array}$ & 19223 (83.1) & 19416 (84.4) & 19381 (84.4) & 19233 (83.9) \\
\hline
\end{tabular}

*Hypertension, diabetes mellitus, dyslipidaemia, arthritis.

†Asthma, allergic rhinitis, atopic dermatitis.

considered for the unequal probability of sampling and non-response.

Descriptive analyses were conducted to describe the participants in 2013, 2015, 2017 and 2019. The logic model with KAP theory of handwashing-related outcomes was examined using the Rao-Scott modified $\chi^{2}$ tests to identify any differences between outcomes in each year. The Rao-Scott modified $\chi^{2}$ is recommended since it yields a more conservative interpretation than the Wald $\chi^{2}$ in population-based complex sample design such as National Health and Nutrition Examination Survey. ${ }^{21}$ Lastly, multivariate logistic regression analysis was applied to assess the association between handwashing-related outcomes and sociodemographic factors, and health status. In the regression modelling, we piled up 4 years of CHS data as one dataset with survey year indication for differences between years and fitted the survey year variable while estimating the relevant covariates.

The first step in logistic regression, we figured out the relationship between EXEP and other factors. Afterwards, we repeated multivariate logistic regression according to logic model to reveal how EXEP is related to HWP: how the EXEP is related to AEHW, and then the AEHW with HWP. Finally, we evaluated whether AEHW is mediating the relation between EXEP and HWP. According to the steps for mediation by Baron and Kenny, we examined the following four conditions to define the AEHW as a mediator between the EXEP and the HWP: (a) the relationship between the EXEP and the HWP should be significant, (b) the relationship between the EXEP and the AEHW should be significant, (c) the relationship between the AEHW and the HWP should be significant 
and (d) in the model adjusted with AEHW, the effect of EXEP to HWP should be smaller than the effect in the model not adjusted with AEHW. ${ }^{22}$ Evaluations of a mediator were approached from crude relations fitted only relevant variables (EXEP, AEHW and HWP), and a survey year variable to a logistic regression model. ${ }^{23}$ Afterwards, the adjusted mediate effect was estimated with adjustment including sociodemographic factors and health conditions. All results were presented as ORs with 95\% CIs. All procedures were conducted using SAS V.9.4 (SAS Institute, Cary, North Carolina, USA) and all statistical tests were two-sided and a $p$ value $<0.05$ was considered to be statistically significant.

\section{Patient and public involvement}

Patients or the public were not involved in this study.

\section{RESULTS}

\section{Demographic characteristics and descriptive statistics}

Annually, total participants were 23139 (men 10 231, 44.2\%), 23004 (men 10 218, 44.4\%), 22955 (men 10 278, 44.8\%) and 22930 (men $10064,43.9 \%$ ) in the years 2013, 2015, 2017 and 2019, respectively (table 1). The overall distributions of participants by sociodemographics were similar over the 4 years. However, the prevalence of comorbidity with chronic conditions was increasing slightly, while those having poor oral health status was decreasing.

The participants who had EXEP were 14922 (64.5\%), $18491(80.4 \%), 16141(70.3 \%)$ and $15538(67.8 \%)$ in each year and AEHW for helpful were 22929 (99.1\%), 22 662 (98.5), 22791 (99.3) and 22753 (99.2\%). Finally practice with soap or hand detergents were 19223 (83.1\%), 19416 (84.4\%), 19381 (84.4\%) and 19233 (83.9\%) for biennially surveyed results (table 1 ).

\section{Logic model in responses}

In order to understand the stepwise relevance between knowledge, attitude and practice, we set the logic model with a hierarchy among the handwashing-related variables. Then we analysed statistically each variable (figure 1).

Frequency regarding EXEP, AEHW and HWP with soap or detergents were presented in table 2. The distribution of AEHW was significantly differentiated according to the EXEP regardless of the survey year. In addition, practice with soap or hand detergents was different depending on the status of AEHW except for 2013. In summary, positive AEHW was higher in the group of 'yes' of EXEP, and good practice was shown more in the positive AEHW.

EXEP prevalence according to adjusting variables including demographics, health status and a survey year, and associations with AEHW and HWP was presented (table 3). EXEP on proper handwashing is more likely to be women (1.36, 95\% CI 1.34 to 1.38) and the populations aged between the 30s and 60s. Higher education levels positively increased the EXEP. Especially participants with college or higher level of education were prominently associated (1.47, 95\% CI 1.43 to 1.51 ).

The EXEP is positively associated with HWP (1.21, 95\% CI 1.19 to 1.23$)$ and AEHW (1.50, 95\% CI 1.38 to $1.63)$ in regression models having EXEP as an explanatory variable, and HWP and AEHW as a response variable separately. Additionally, AEHW was shown the increased association with HWP (1.67, 95\% CI 1.53 to 1.81$)$ in the demographic variables adjusted model. In summary, according to the models based on the logic model, women rather than men and participants with higher education levels and good self-rated health or oral health conditions were more likely to accommodate with the knowledge and attitudes in an ideal direction.

\section{Attitude as mediator between exposure to educational events} and practice in handwashing

Apart from the logic model, we considered AEHW as a mediate variable and compared the effect size of EXEP with HWP. Using two separate regression models, direct and indirect effects were estimated separately. The crude association among EXEP, AEHW and HWP, the direct effect was EXEP (1.33, 95\% CI 1.31 to 1.36$)$ to HWP controlled by AEHW and the crude indirect effect could be calculated by multiplying AEHW (1.76, 95\% CI 1.62 to 1.91) to HWP controlled by EXEP and EXEP (1.69, 95\% CI 1.56 to 1.83$)$ to AEHW. The adjusted associations considering sociodemographic factors and health status, the direct was $(1.21,95 \%$ CI 1.18 to 1.23$)$ and the indirect effect could be obtained by multiplying AEHW (1.62, 95\% CI 1.48 to 1.76$)$ and EXEP $(1.50,95 \%$ CI 1.38 to 1.63$)$. The associations between EXEP and HWP were $(1.21,95 \%$ CI 1.18 to 1.23$)$ and $(1.21,95 \%$ CI 1.19 to 1.23$)$ with and without controlled by AEHW, respectively in adjusted regression models. The mediated effect of AEHW to HWP is not determined evidently because the effect size of EXEP was not comparatively differentiated between with and without AEHW as a mediate variable (figure 2).

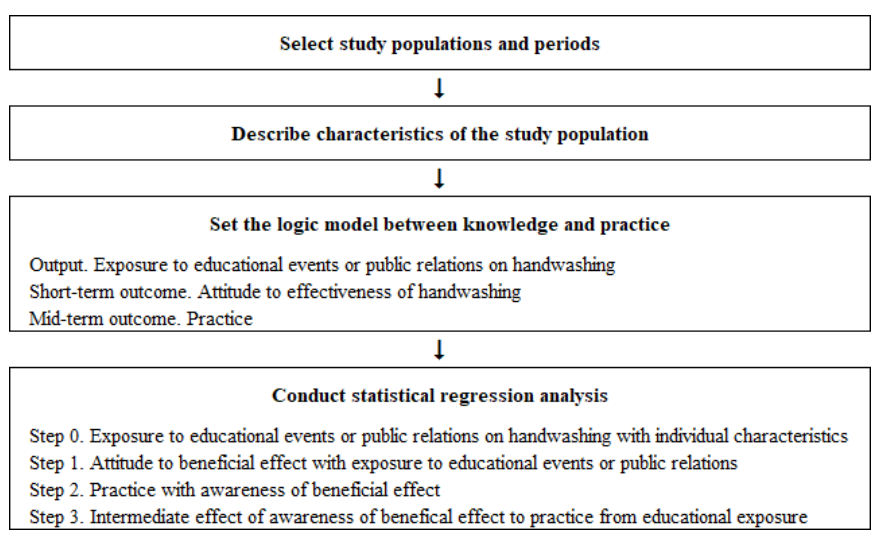

Figure 1 Logic model and statistical analysis for public relations with handwashing, awareness of beneficial effect and handwashing practice. 


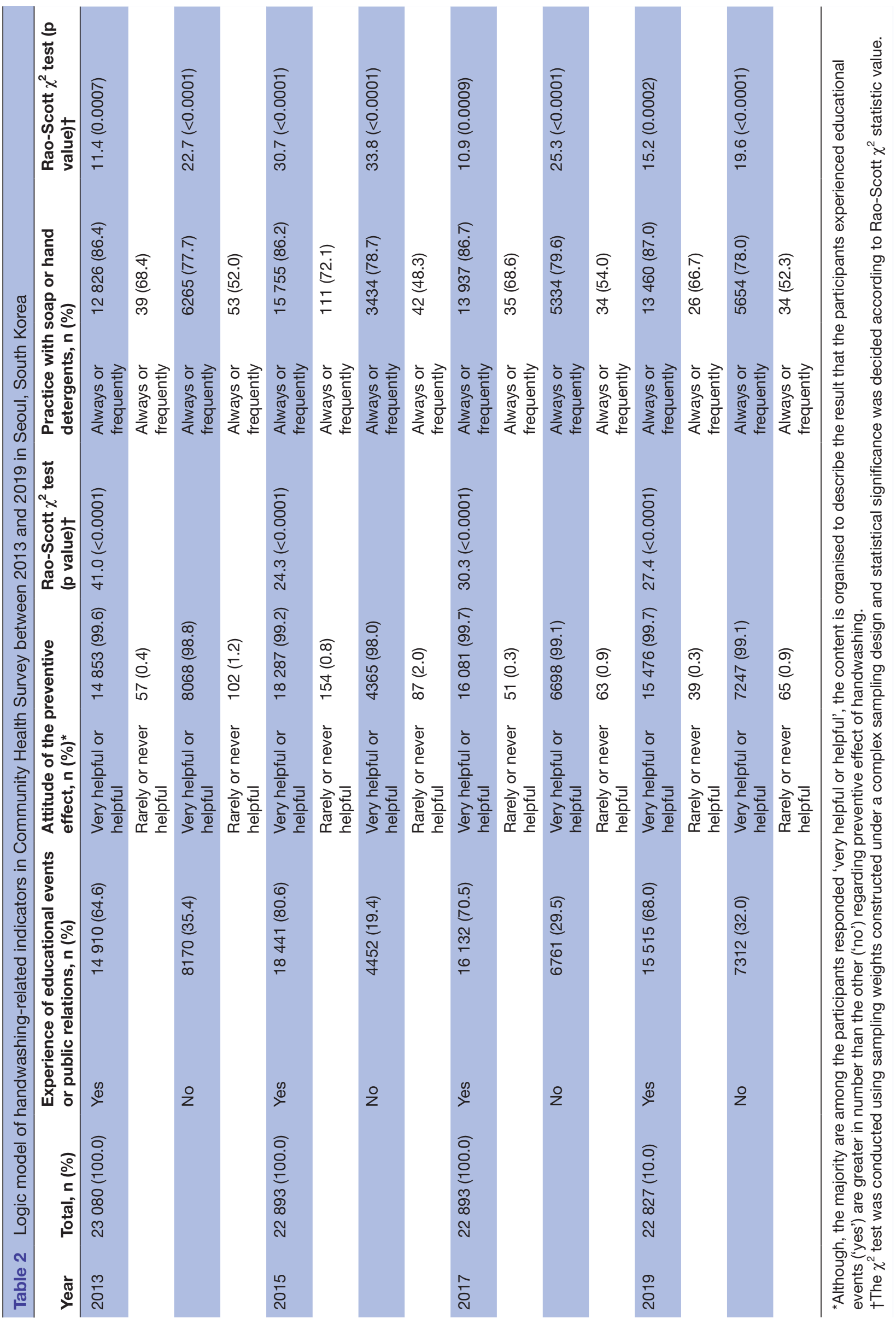




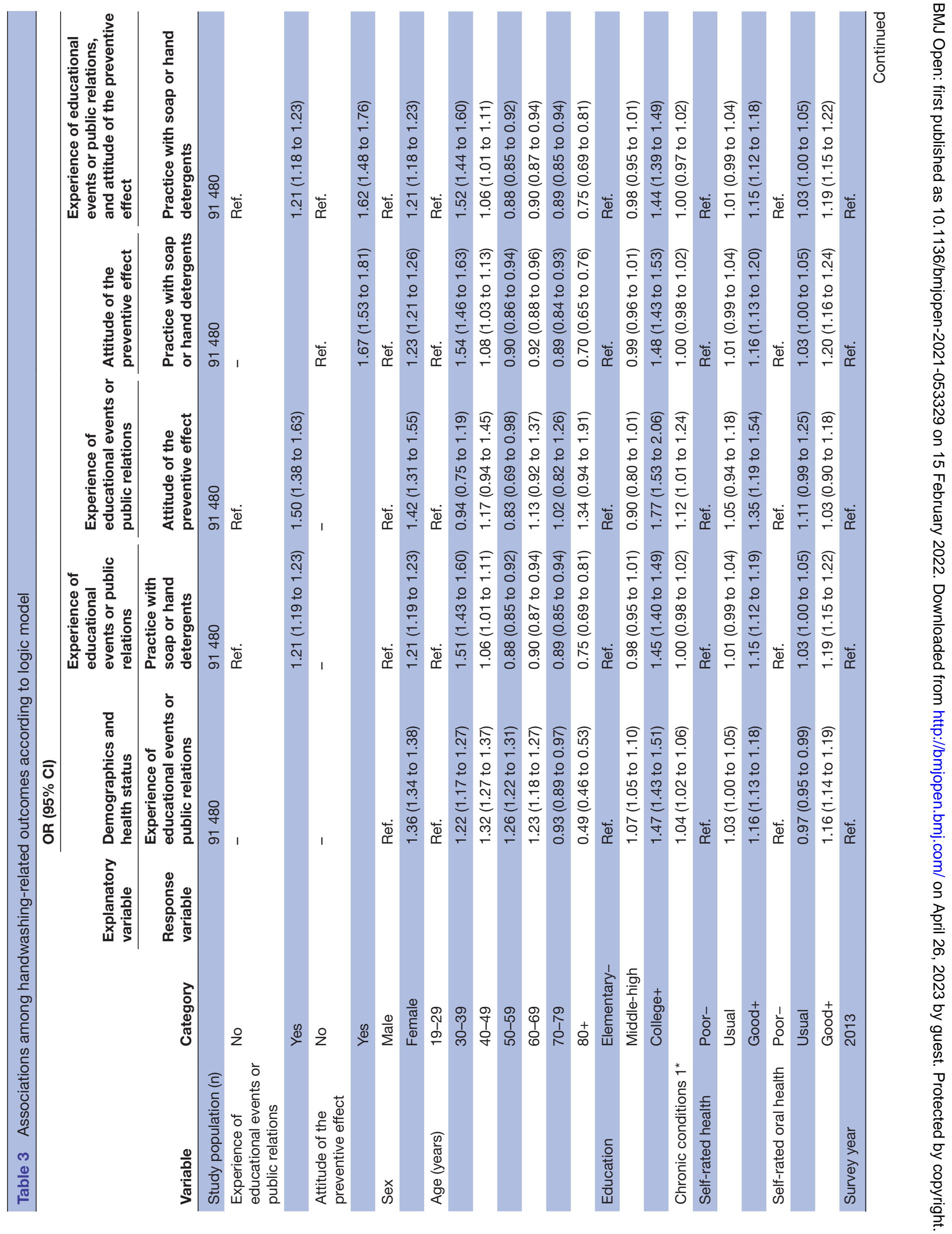



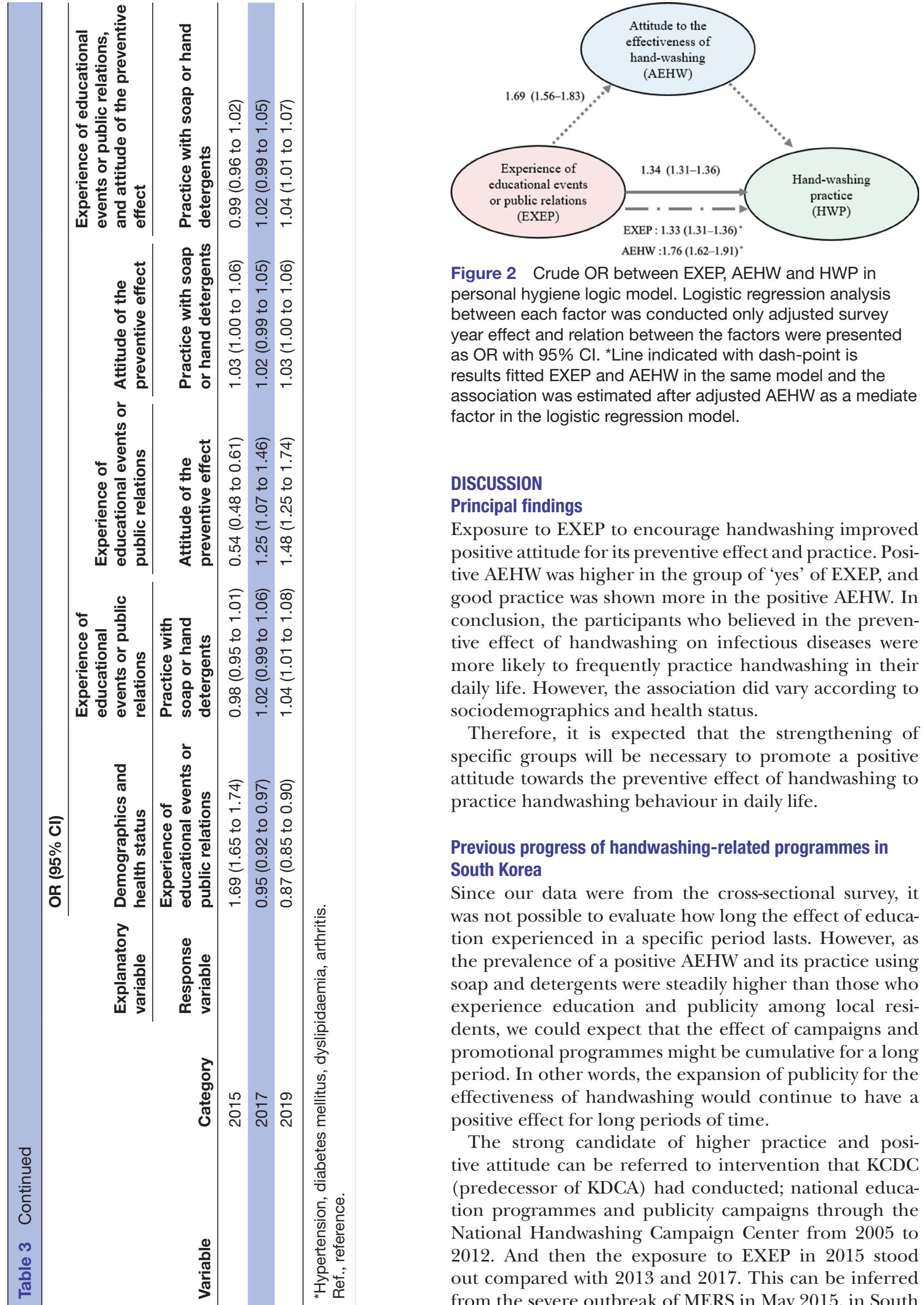

Figure 2 Crude OR between EXEP, AEHW and HWP in personal hygiene logic model. Logistic regression analysis between each factor was conducted only adjusted survey year effect and relation between the factors were presented as OR with $95 \% \mathrm{Cl}$. "Line indicated with dash-point is results fitted EXEP and AEHW in the same model and the association was estimated after adjusted AEHW as a mediate factor in the logistic regression model.

\section{DISCUSSION}

\section{Principal findings}

Exposure to EXEP to encourage handwashing improved positive attitude for its preventive effect and practice. Positive AEHW was higher in the group of 'yes' of EXEP, and good practice was shown more in the positive AEHW. In conclusion, the participants who believed in the preventive effect of handwashing on infectious diseases were more likely to frequently practice handwashing in their daily life. However, the association did vary according to sociodemographics and health status.

Therefore, it is expected that the strengthening of specific groups will be necessary to promote a positive attitude towards the preventive effect of handwashing to practice handwashing behaviour in daily life.

\section{Previous progress of handwashing-related programmes in South Korea}

Since our data were from the cross-sectional survey, it was not possible to evaluate how long the effect of education experienced in a specific period lasts. However, as the prevalence of a positive AEHW and its practice using soap and detergents were steadily higher than those who experience education and publicity among local residents, we could expect that the effect of campaigns and promotional programmes might be cumulative for a long period. In other words, the expansion of publicity for the effectiveness of handwashing would continue to have a positive effect for long periods of time.

The strong candidate of higher practice and positive attitude can be referred to intervention that KCDC (predecessor of KDCA) had conducted; national education programmes and publicity campaigns through the National Handwashing Campaign Center from 2005 to 2012. And then the exposure to EXEP in 2015 stood out compared with 2013 and 2017. This can be inferred from the severe outbreak of MERS in May 2015, in South 
Korea $^{24}$ and the impact of the resulting quantitative expansion of public relations for health promotion. ${ }^{13}$

\section{Literature reviews with related study results}

Women showed more association versus men and varied according to age groups. Several studies have shown that the degree of acceptability of certain information is higher for women than for men. ${ }^{2526}$ Similar results were suggested in a comparison of perception between men and women, who are distinct demographics and health status. ${ }^{27}$ Our study also showed attitudes that are more positive in the younger age group (online supplemental table S1). These results are in line with other studies that showed differences in risk perception according to age. ${ }^{28}$ On the other hand, several studies have shown that older adults had better risk perception than younger adults. ${ }^{29} 30$

In terms of education level, the positive AEHW was stronger for the subjects with college or higher level. More positive attitude with a higher education level was found in various studies. ${ }^{131}$ It seems that the perception and compliance of health information were better as the level of education was higher. This might be explained by the assumption that those with higher education levels would have a higher probability to ascertain something is beneficial among an overwhelming amount of information promoting tactics to improve health.

Regarding comorbidity conditions, participants with chronic conditions such as hypertension, diabetes mellitus, dyslipidaemia and arthritis were more likely to practice proper handwashing when they have positive attitude towards the preventive effect of handwashing. In our study, presence of comorbidity is within a recent year which is same period with EXEP. Although the result does not explain the relationship between comorbidity conditions and positive attitude towards preventive effect is not fully explainable with only survey results, majority with comorbidity are more likely to pay attention to lifestyles that prevent health risks.

In addition, participants with good subjective health and good oral health status were more likely to practice handwashing and have a positive attitude towards preventive effect by practicing handwashing. For instance, participants with a positive attitude in handwashing were more likely to practice desirable handwashing in daily life. The increased association was shown for good subjective health $(1.16,95 \%$ CI 1.13 to 1.20$)$ and good oral health (1.20, 95\% CI 1.16 to 1.24$)$ by comparison with poor health participants.

In a recent study for the general population of Hong Kong, there was no difference in knowledge of handwashing according to self-rated health status as a result of multivariate regression analysis. ${ }^{32}$ However, our study showed significant differences in several models (table 3).

There were the days when it was expected that the threat of infectious diseases would soon disappear with the contribution of antibiotics and vaccines. However, our expectations have already collapsed with the continued emerging issues of infectious diseases: HIV and AIDS,
SARS, MERS, animal infections such as avian influenza, foot and mouth disease as well as zoonosis such as swine influenza, Spanish influenza, Ebola haemorrhagic fever. Still, infectious diseases remain an area to struggle with for human beings. Most infectious diseases could be preventive with thorough handwashing. ${ }^{12}$

Lastly, we set the logic model with a hierarchy among handwashing-related variables and compared the effect size and statistical significance. The AEHW was analysed as a mediate variable in the model, but the effect size with or without the mediator was shown similar, although other prior conditions met the criteria of a traditional approach to mediation. These results can be explained by the fact that participants with good attitudes toward the preventive effect maintained the attitudes acquired through educational experience prior to last year for a much longer period of time. According to the proportion table, the participants with a better attitude to the effectiveness of handwashing were much higher than those with EXEP within the recent 1 year. Therefore, the potential mediation might be latent and not perform its role in the observational study design.

\section{Study limitations}

Our study has some limitations. First, the questionnaire recruited to define EXEP was not designed to verify educational events and public relations individually. A recruited questionnaire to define the EXEP includes both aspects of educational events and public relations. In general, education has a specified and driven purpose and induce subjects to meet the acquisitions of educational performance. Public Relations, on the other hand, aims to disseminate knowledge to the public with relatively low motivation compared to education. Applying the KAP theory, EXEP was used as a term of knowledge to change the attitude regarding self-handwashing. Therefore, the spectrum and depth of giving knowledge to the subjects using the questionnaire are vague in this study.

Second, it was impossible to quantify the handwashing publicity by year or in what way. We cannot understand how each method influenced the perception or actual behaviour of handwashing, because the health promotion activity based on the community was varied in the form and method in each region.

Publicity measures and education were disseminated about various preventive methods during the MERS pandemic in early 2015, in South Korea. However, how much information the population could accept and the resultant positive attitude were not estimable. Nevertheless, by linking 4 years of proportions to EXEP linearly, we presume there was additional exposure to EXEP in 2015.

Third, using one question under retrospective study design to identify the AEHW to prevent infectious diseases is limited to measuring and verifying the populations' distributed variations. Regarding the subjective question, almost all participants responded 'yes' is hard to mean that 'yes' participants' attitudes are all at the same level with confidence. The study design based on 
a survey-based ecological approach seems hard to find strong evidence relatively and explain association due to report bias.

Lastly, it is not possible to identify the differences in the method of analysing whether the subjects are well informed about the frequency or the application of personal hygiene properly or roughly applied the actual behaviour. In other words, quantitative behaviour of recognition through handwashing promotion can be estimated, but it is not possible to grasp qualitative behaviour in application because of the limitations of survey items in this study.

\section{Conclusions}

The community-based epidemiological investigation on exposure to giving knowledge of handwashing and behavioural outcomes provided that participants who believed in the preventive effect of handwashing on infectious diseases were more likely to practice handwashing frequently in their daily life. Furthermore, the delivery of health information to the public can achieve ultimate outcomes when the targets' positive attitude towards the informed effectiveness is formed. Although the effects of the handwashing-related variables in the logic model with hierarchy were not strongly distinguishable, this study implicates that taking knowledge is more likely to lead to behavioural change only when it works to form a positive attitude towards its effect.

Contributors Conceptualisation: JK, KY. Data curation: JK. Formal analysis: JK. Funding acquisition: JK. Methodology: JK, KY. Project administration: JK. Visualisation: JK. Writing —original draft: JK, KY. Writing—review and editing: JK, $\mathrm{KY}$. Responsible for the overall content as the guarantor: $\mathrm{KY}$

Funding This work was supported by the National Research Foundation of Korea (NRF) grant funded by the Korean government (MSIP) (no. 2017R1C1B2002663).

Competing interests None declared.

Patient consent for publication Not applicable.

Ethics approval This study involves human participants but IRB No.

E1707/003-010 exempted this study. Participants gave informed consent to participate in the study before taking part.

Provenance and peer review Not commissioned; externally peer reviewed.

Data availability statement Data are available in a public, open access repository. The data and other additional about CHS data are available in the following website of Community Health Survey: https://chs.kdca.go.kr/chs/rdr/rdrlnfoProcessMain.do.

Supplemental material This content has been supplied by the author(s). It has not been vetted by BMJ Publishing Group Limited (BMJ) and may not have been peer-reviewed. Any opinions or recommendations discussed are solely those of the author(s) and are not endorsed by BMJ. BMJ disclaims all liability and responsibility arising from any reliance placed on the content. Where the content includes any translated material, BMJ does not warrant the accuracy and reliability of the translations (including but not limited to local regulations, clinical guidelines, terminology, drug names and drug dosages), and is not responsible for any error and/or omissions arising from translation and adaptation or otherwise.

Open access This is an open access article distributed in accordance with the Creative Commons Attribution Non Commercial (CC BY-NC 4.0) license, which permits others to distribute, remix, adapt, build upon this work non-commercially, and license their derivative works on different terms, provided the original work is properly cited, appropriate credit is given, any changes made indicated, and the use is non-commercial. See: http://creativecommons.org/licenses/by-nc/4.0/.

\section{ORCID iDs}

Jayeun Kim http://orcid.org/0000-0002-8538-7843
Kyuhyun Yoon http://orcid.org/0000-0001-7017-1145

\section{REFERENCES}

1 Rabbi SE, Dey NC. Exploring the gap between hand washing knowledge and practices in Bangladesh: a cross-sectional comparative study. BMC Public Health 2013;13:89.

2 Najnin N, Leder K, Qadri F, et al. Impact of adding hand-washing and water disinfection promotion to oral cholera vaccination on diarrhoea-associated hospitalization in Dhaka, Bangladesh: evidence from a cluster randomized control trial. Int J Epidemiol 2017;46:2056-66.

3 World Health Organization. Who guidelines on hand hygiene in health care: first global patient safety challenge clean care is safer care, 2009. Available: https://pubmed.ncbi.nlm.nih.gov/23805438/

4 Joshi SK, Joshi A, Park BJ, et al. Hand washing practice among health care workers in a teaching hospital. J Nepal Health Res Counc 2013;11:1-5.

5 Oh HS, Knowledge OHS. Knowledge, perception, performance, and attitude regarding hand hygiene and related factors among infection control nurses in South Korea: a cross-sectional study. Am J Infect Control 2019;47:258-63.

6 Little P, Stuart B, Hobbs FDR, et al. An internet-delivered handwashing intervention to modify influenza-like illness and respiratory infection transmission (PRIMIT): a primary care randomised trial. Lancet 2015;386:1631-9.

7 Jeong JS, Choi JK, Jeong IS, et al. [A nationwide survey on the hand washing behavior and awareness]. J Prev Med Public Health 2007;40:197-204.

8 Lee M-S, Hong SJ, Kim Y-T. Handwashing with soap and national handwashing projects in Korea: focus on the National handwashing survey, 2006-2014. Epidemiol Health 2015;37:e2015039-e39.

9 KCDC. H1N1 influenza promotional materials, 2013. Available: http:// www.cdc.go.kr/gallery.es? mid=a20503020000\&bid=0003 [Accessed 09 Aug 2019].

10 KCDC. Knowing MERS immediately and how to prevent infections from travelers in middle East countries (6 languages), 2018. Available: http://www.cdc.go.kr/gallery.es?mid=a20503020000\&bid= 0003 [Accessed 09 Aug 2019].

11 KCDC. Mers prevention guidlines (domestic, foreign), 2018. Available: http://www.cdc.go.kr/gallery.es?mid=a20503020000\&bid= 0003 [Accessed 09 Aug 2019].

12 World Health Organization. Infection prevention and control in health care for confirmed or suspected cases of pandemic (H1N1) 2009 and influenza-like illnesses, 2010. Available: https://www.who.int/csr/ resources/publications/cp150_2009_1612_ipc_interim_guidance_ h1n1.pdf

13 Korean Centers for Disease Control \& Prevention (KCDC). Status and Prevention of Middle East Respiratory Syndrome South Korea, 2018. Available: http://www.cdc.go.kr/CDC/info/CdcKrlnfo0301.jsp? menulds=HOME006-MNU3003-MNU2950-MNU2951\&cid=26688 [Accessed 19 Dec 2018].

14 Taware S, Gawai P, Chatterjee A, et al. Outcome of school-based intervention program in promoting personal hygiene in primary school children of Mumbai, India. Int Q Community Health Educ 2018;39:31-8.

15 Paul KK, Panigrahi SK, Soodi Reddy AK, et al. Association of personal hygiene with common morbidities among upper primary school children in rural Odisha. J Family Med Prim Care 2017;6:509-12.

16 Khatoon R, Sachan B, Khan MA, et al. Impact of school health education program on personal hygiene among school children of Lucknow district. J Family Med Prim Care 2017;6:97-100.

17 Sreenivasan N, Gotestrand SA, Ombeki S, et al. Evaluation of the impact of a simple hand-washing and water-treatment intervention in rural health facilities on hygiene knowledge and reported behaviours of health workers and their clients, Nyanza Province, Kenya, 2008. Epidemiol Infect 2015;143:873-80.

18 Lee M, Kang B-A, You M. Knowledge, attitudes, and practices (KAP) toward COVID-19: a cross-sectional study in South Korea. BMC Public Health 2021;21:295.

19 Raina S. Assessment of knowledge, attitude, and practice in health care delivery. N Am J Med Sci 2013;5:249-50.

20 Korea Disease Control and Prevention Agency, Bureau of Chronic Disease Prevention and Control, Division of Chronic Disease Control. Community health survey data, 2019. Available: https://chs.kdca.go. $\mathrm{kr} / \mathrm{chs} / \mathrm{rdr} / \mathrm{rdrlnfoProcessMain.do}$

21 JNK R, Scott AJ. On simple adjustments to chi-square tests with sample survey data. Ann Stat 1987;15:385-97. 
22 Baron RM, Kenny DA. The moderator-mediator variable distinction in social psychological research: conceptual, strategic, and statistical considerations. J Pers Soc Psychol 1986;51:1173-82.

23 Rijnhart JJM, Twisk JWR, Eekhout I, et al. Comparison of logisticregression based methods for simple mediation analysis with a dichotomous outcome variable. BMC Med Res Methodol 2019;19:19.

$24 \mathrm{Kim} \mathrm{KH}$, Tandi TE, Choi JW, et al. Middle East respiratory syndrome coronavirus (MERS-CoV) outbreak in South Korea, 2015: epidemiology, characteristics and public health implications. $J$ Hosp Infect 2017;95:207-13.

25 Yang J, Park E-C, Lee SA, et al. Associations between hand hygiene education and self-reported hand-washing behaviors among Korean adults during MERS-CoV outbreak. Health Educ Behav 2019;46:1090198118783829.

26 Al-Rifaai JM, Al Haddad AM, Qasem JA. Personal hygiene among college students in Kuwait: a health promotion perspective. J Educ Health Promot 2018;7:92.

27 Bai Y, Leib AY, Puri AM, et al. Gender differences in crowd perception. Front Psychol 2015;6:1300.
28 Bonem EM, Ellsworth PC, Gonzalez R. Age differences in risk: perceptions, intentions and domains. J Behav Decis Mak 2015;28:317-30.

29 Rhodes N, Pivik K. Age and gender differences in risky driving: the roles of positive affect and risk perception. Accid Anal Prev 2011;43:923-31.

30 Kaba Z, Khamisa N, Tshuma N. Age-Group differences in risk perceptions of non-communicable diseases among adults in Diepsloot township, Johannesburg, South Africa: a cross-sectional study based on the health belief model. S Afr Med J 2017;107:797.

31 Pensiero N, Schoon I. Social inequalities in educational attainment: The changing impact of parents' social class, social status, education and family income, England 1986 and 2010. Longit Life Course Stud 2019;10:87-108.

32 Suen LKP, So ZYY, Yeung SKW, et al. Epidemiological investigation on hand hygiene knowledge and behaviour: a cross-sectional study on gender disparity. BMC Public Health 2019;19:401. 